Research Paper

\title{
The Predictive and Prognostic Role of Stromal Tumor-infiltrating Lymphocytes in HER2-positive Breast Cancer with Trastuzumab-based Treatment: a Meta-analysis and Systematic Review
}

\author{
Tao Xu1*, Bang-Shun He ${ }^{1 *}$, Xiang-Xiang Liu ${ }^{1}$, Xiu-Xiu Hu${ }^{1}$, ${ }^{2}$ Kang Lin ${ }^{1}$, Yu-Qin Pan ${ }^{1}$, Hui-Ling Sun ${ }^{1}$, \\ Hong-Xin Peng ${ }^{1,2}$, Xiao-Xiang Chen ${ }^{1,2}$, Shu-Kui Wang ${ }^{1 凶}$ \\ 1. General Clinical Research Center, Nanjing First Hospital, Nanjing Medical University, Nanjing, China; \\ 2. Medical college, Southeast University, Nanjing, China. \\ * These authors contributed equally to this work. \\ $\triangle$ Corresponding author: Shu-Kui Wang, e-mail: sk_wang@njmu.edu.cn \\ (c) Ivyspring International Publisher. This is an open access article distributed under the terms of the Creative Commons Attribution (CC BY-NC) license \\ (https://creativecommons.org/licenses/by-nc/4.0/). See http://ivyspring.com/terms for full terms and conditions.
}

Received: 2017.05.16; Accepted: 2017.08.19; Published: 2017.10.17

\begin{abstract}
Background Tumor-infiltrating lymphocytes (TILs) are white blood cells that have left the bloodstream and migrated into a tumor, involving in the prognosis of breast cancer $(B C)$ patients. Published studies reported the value of TILs in patients with HER2-positive receiving trastuzumab-based treatment. However, the results obtained remain controversial. Here, we conducted this study to explore the predictive and prognostic role of TILs for HER2-positive BC patients receiving trastuzumab therapies.

Method To identify the related published studies, a comprehensive literature search dating up to July 2017 was performed in the databases of PubMed, PMC, Web of Science and China National Knowledge Infrastructure $(\mathrm{CNKI})$ according to predefined selection criteria. The pathologic complete response (PCR) and survival outcome of patients were measured by odds ratio (OR) and hazard ratio (HR) with corresponding $95 \%$ confidence interval $(95 \% \mathrm{Cl})$, respectively. The association between TILs and trastuzumab benefit was analyzed by using STATA version 11.0.

Result Eleven eligible studies comprising 3228 patients were identified in the present study. The pooled results showed that high level of TILs was associated with a significantly improved $\mathrm{PCR}$ rate $(\mathrm{OR}=1.32 ; 95 \% \mathrm{Cl}=$ 1.10-1.60) and longer survival $(\mathrm{HR}=0.97 ; 95 \% \mathrm{Cl}=0.96-0.99)$, particularly in the subgroups of retrospective study design and 10\% INC cut-off value. Moreover, stratified analysis revealed that elevated TILs was a predictor of higher PCR rate in the Asian population and improved survival in the subgroups of Caucasian population and multivariate analysis.

Conclusion This meta-analysis indicated that the level of stromal TILs was an independent predictive and prognostic marker for better outcome in HER2-positive BC patients receiving trastuzumab-based treatment. High level of TILs was significantly associated with trastuzumab benefit.
\end{abstract}

Key words: tumor-infiltrating lymphocytes; breast cancer; trastuzumab; pathologic complete response; survival.

\section{Introduction}

Breast cancer $(\mathrm{BC})$ is recognized as the most common invasive malignant tumor, which accounting for $30 \%$ of all new cancer cases and $14 \%$ of all cancer deaths in women, and it is the second leading cause of cancer-related death among females all over the world [1]. BC is a complex multifactorial disease with high heterogeneity and can be divided into several subtypes according to the molecular markers, such as luminal (the receptors, $\mathrm{ER}^{+}$and/or $\mathrm{PgR}^{+}, \mathrm{HER} 2^{-}$), HER2-positive and triple negative BC (TNBC, these three receptors are all negative expression). HER2 is overexpressed in approximately $20-30 \%$ of newly 
diagnosed breast carcinomas [2, 3] and is considered to be associated with aggressive disease progression, a high distant metastasis rate and an adverse prognosis [2, 4]. Nevertheless, the humanized monoclonal antibody, targeting and blocking the HER2/neu receptor such as trastuzumab, has been applied to clinical treatment of BC patients, and has shown the great improvement of the overall survival in HER2-positive BC patients [5]. However, a significant challenge was presented for the clinical application of these drugs, indicating an increasingly important issue that resistance to anti-HER2 agents such as trastuzumab. Therefore, clinical useful biomarkers are urgently available to identify the HER2-positive patients who derive benefit from or are resistant to trastuzumab.

The tumorigenesis of $\mathrm{BC}$ was determined by several factors, such as individuals' genetic alteration, phenotypic transformation of cancer cells and interaction between the immunological microenvironment and malignant tumor cells, which also contributed to the progression of BC. As important components of microenvironment, tumor-infiltrating lymphocytes (TILs) are a group of cells comprising the majority of mononuclear immune infiltrates from the innate and adaptive immune response, participating in killing tumor cells and reflecting an individual immunological tumor response [6]. Tumor cells could inactivate or exhaust TILs through direct suppression of antitumor immune cells (such as $\mathrm{CD}^{+} \mathrm{T}$ helper 1 (Th1) cells, CD8 ${ }^{+}$ cytotoxic $\mathrm{T}$ cells, NK cells and macrophages) or recruitment and reactivation of immunosuppressive subsets (such as $\mathrm{CD}^{+}$forkhead box $\mathrm{P} 3\left(\mathrm{FOXP3}^{+}\right.$), $\mathrm{CD}^{+}$Th2 cells, myeloid-derived suppressor cells (MDSCs)) leading to diminishing host antitumor immune responses [7, 8]. Although $\mathrm{BC}$ has not traditionally been considered as an immunogenic disease, studies have confirmed that the presence of immunological components such as TILs in BC was associated with higher therapeutic responses to immunogenic chemotherapy $[7,9,10]$, which is predictive of prognosis and benefit from chemotherapy in the neoadjuvant and adjuvant setting $[9,11]$. Moreover, accumulated clinical trials have demonstrated that TILs were associated with improved outcome of BC patients [11-15]. These evidences indicated that the efficacy of some cancer therapies was dependent on the creation of a favorable immune microenvironment $[16,17]$, which was also observed in the patients receiving the monoclonal antibody treatment [18]. Trastuzumab, as the standard of care for HER2-amplified BC, exerts complete therapeutic effects relating to contribution of functional innate and adaptive immunity $[19,20]$.
The potential mechanism of the association between TILs and trastuzumab benefit is that the exhausted immune response and intratumoral immunosuppression might be relieved by trastuzumab and in turn promoting antitumor immune response may enhance the efficacy of trastuzumab [7, 21]. Loi S. et al. firstly reported that each $10 \%$ increase of TILs was significantly associated with decreased distant recurrence in HER2-positive BC patients after treatment with trastuzumab [22]. Additionally, the predictive role of TILs on pathological complete response (pCR) in $\mathrm{HER}^{+} \mathrm{BC}$ patients receiving neoadjuvant containing trastuzumab was demonstrated a significant association [23].

Although it is accepted that high level of TILs in $\mathrm{BC}$ may have predictive value of $\mathrm{pCR}$ in neoadjuvant setting and good survival in adjuvant setting, especially in TNBC [24-28], the role of TILs among patients bearing HER2 overexpression still remains obscure. Some studies reported that high level of TILs was associated with trastuzumab benefit [23, 29-33], while others showed that elevated TILs did not significantly predict for trastuzumab benefit [22, 34-37]. In order to comprehensively evaluate the association between TILs and trastuzumab benefit, we performed the current meta-analysis.

\section{Methods}

\section{Search strategy}

This systematic review and meta-analysis were conducted according to the Preferred Reporting Items for Systematic Reviews and Meta-Analyses (PRISMA) statement and methods [38, 39]. We identified studies of interest by conducting an electronic literature search in the databases of PubMed, PMC, Web of Science and China National Knowledge Infrastructure (CNKI) before July 2017 and using various combinations of the following key words: 'breast cancer or tumor or carcinoma or neoplasm', 'HER2and/or HER-2 positive', 'tumor- or tumour-infiltrating lymphocytes', 'pathologic complete response $(\mathrm{pCR})^{\prime}$, ' prediction or prognosis or survival'. Additionally, in order to avoid omitting related literature, some cited references in relevant studies including review articles were all manually searched for additional eligible papers.

\section{Selection criteria}

We selected articles that evaluated the benefit of trastuzumab in HER2-positive BC where $\mathrm{pCR}$ and outcome measures were compared in different levels of TILs at diagnosis. All search results met the following criteria: (1) clinical trials or cohorts on human beings; (2) patients diagnosed with 
HER2-positive BC and received trastuzumab-based treatment in the neoadjuvant or adjuvant setting; (3) investigated the predictive or prognostic role of TILs; (4) sufficient data provided for the odds ratio (OR) with $95 \%$ confidence interval $(95 \% \mathrm{CI})$ for $\mathrm{pCR}$ or hazard ratio (HR) with 95\% CI for survival outcomes; (5) published as original articles and the language of publication was restricted to English and Chinese. Also, the types of reviews, comments or letters and studies with duplicate data or incomplete information were excluded from this analysis.

\section{Data extraction}

Data from all eligible publications were extracted according to the inclusion criteria by two independent researchers (Tao $\mathrm{Xu}$ and Xiang-xiang Liu). Any discrepancies were resolved through discussion with a third reviewer (Bang-shun $\mathrm{He}$ ) or referring back to the original articles until the two reviewers reached a consensus. The following information was collected: the first author, published years, study design, the number of patients, country of origin, ethnicity of the study population, TILs cut-off value, TILs detection method, duration of follow-up, pCR definition, outcome measures, use of univariate or multivariate logistic model analysis and treatment regimens.

The outcome measures extracted from these selected studies were $\mathrm{pCR}$, disease-free survival (DFS), distant disease-free survival (DDFS), event-free survival (EFS), recurrence-free survival (RFS), progression-free survival (PFS). pCR was defined as the absence of residual invasive cancer cells in breast. DFS was defined as the time from randomization to the recurrence of breast cancer at any site. DDFS was defined as the time interval between the date of randomization and the date of first cancer recurrence outside of the ipsilateral locoregional region or to death whenever death occurred before distant recurrence. EFS was defined as the time from randomization to first event, including recurrence, disease relapse, distant metastasis, or death from any cause. RFS was defined as the time from randomization until recurrence of breast cancer. PFS was defined as the time from randomization to the first evidence of progressive disease or death from any cause. Considering all outcome events based on the development of breast cancer and EFS contained every other study endpoint, we decided to choose EFS as a uniform standard for prognostic outcome. Additionally, when different cut-off values were used to define for high and low levels for TILs in one article, we selected the frequently used in most articles and extracted the corresponding OR or HR for pCR or survival outcome.

\section{Statistical analysis}

The association between TILs and trastuzumab benefit in neoadjuvant and adjuvant setting was presented by pooled the OR or HR and its corresponding 95\% CIs. Cochran's Q test and Higgins I-squared were used to assess the heterogeneity between studies [40,41], and subgroup analysis was further conducted to explore the sources of heterogeneity. The random-effects model (DerSimonian-Laird method) [42] was performed in the presence of significant heterogeneity $\left(p<0.05\right.$ or $I^{2}$ $>50 \%$ while the fixed-effects model (Mantel-Haenszel method) [43] was used in its absence $\left(p>0.05\right.$ or $\left.I^{2}<50 \%\right)$. Sensitivity analysis was conducted to examine the stability of the pooled results. Publication bias was assessed by the funnel plot with the Egger's and Begg's tests [44, 45]. All statistical analyses were carried out by STATA version 11.0 (Stata Corporation, College Station, TX, USA).

\section{Results}

\section{Search results}

A total of 586 records were identified after a primary literature search according to the above search strategy and the flow chart of eligible articles selection was presented in Figure 1. Finally, eleven articles comprising 3228 patients were enrolled in this study [22, 23, 29-37] after exclusion of duplicates, irrelevance and articles that did not meet the inclusion criteria. Among these articles, seven studies assessed survival outcomes [22, 30-32, 34-36] and six studies assessed pCR [23, 29, 30, 32, 33, 37], respectively.

\section{Study characteristics}

The characteristics of included studies are summarized in Table 1. The study design of eligible articles was retrospective $(n=7)$, prospective $(n=2)$ and prospective-retrospective $(n=2)$. These articles were published from 2014 to 2017 and each study enrolled the number of patients ranging from 32 to 678 . Ethnicity of patients in these studies included Caucasian $(n=5)$, Asian $(n=3)$ and Mix $(n=3)$. Almost all of the studies adopted H\&E staining method to assess quantities of TILs and only one study used IHC method [33]. The most common cut-off value used for TILs was $10 \%$ increment $(10 \%$ INC) $(n=6)$. The available follow-up time was long enough for the outcomes to occur. The definition of $\mathrm{pCR}$ in all studies was the absence of residual invasive tumor cells in breast and lymph nodes (ypTOis ypN0). Multivariate analysis was conducted to obtain the useful data in eight studies and univariate analysis in two studies, respectively. The trastuzumab-based therapy in 
combination with traditional chemotherapy was applied in each study. Six neoadjuvant studies reported the association between TILs and PCR of the HER2-positive BC patients who received trastuzumab-based neoadjuvant therapy.
Additionally, TILs as a prognostic factor was evaluated in five studies based on adjuvant setting and two studies based on neoadjuvant setting, respectively.

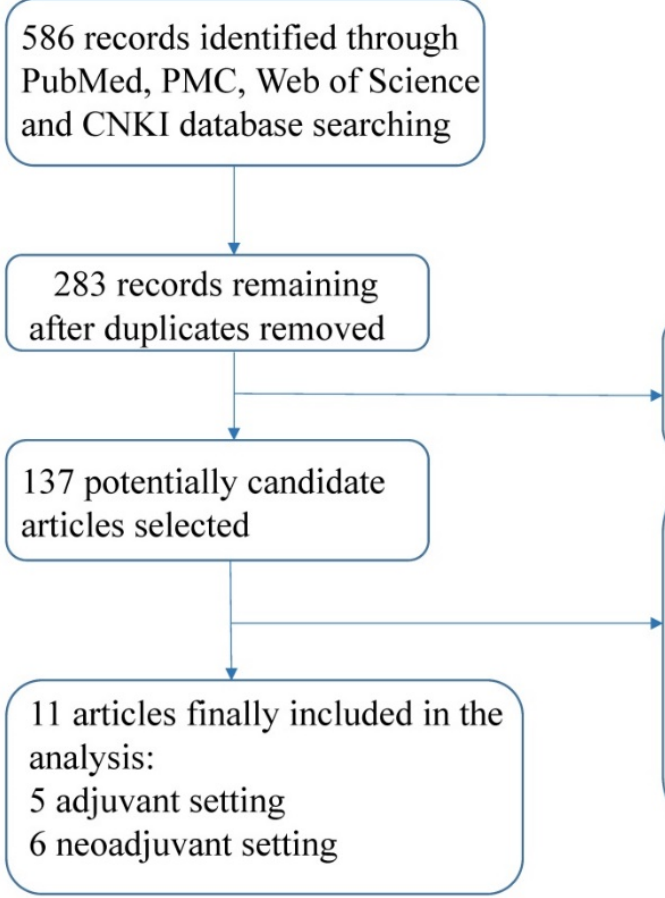

146 records excluded due to irrelevance after screening title and abstract

126 articles excluded after full-text review 69 reviews, letters or comments 9 not human researches 20 fundamental experiment study 24 not HER2 + BC or without Trastuzumab treatment 4 insufficient information

Figure 1. Flow chart of study selection.

Table 1. Baseline Characteristics of the eligible studies

\begin{tabular}{|c|c|c|c|c|c|c|c|c|c|c|c|c|c|}
\hline Author & Year & Study design & $\begin{array}{l}\text { No. of } \\
\text { patients }\end{array}$ & Country & Ethnicity & $\begin{array}{l}\text { Cut-off } \\
\text { value }\end{array}$ & Method & $\begin{array}{l}\text { Median } \\
\text { follow up }\end{array}$ & $\begin{array}{l}\mathrm{pCR} \\
\text { definition }\end{array}$ & Outcome & Analysis & Treatment & Time \\
\hline Loi et al. & 2014 & prospective-retrospective & 209 & multicenter & Mix & $10 \%$ INC & $\mathrm{H} \& \mathrm{E}$ & $\begin{array}{l}62 \\
\text { months }\end{array}$ & - & DDFS & univariate & $\mathrm{D}, \mathrm{FEC}, \mathrm{H}$ & $\begin{array}{l}\text { Adjuvant } \\
\text { setting }\end{array}$ \\
\hline $\begin{array}{l}\text { Denkert } \\
\text { et al. }\end{array}$ & 2015 & prospective & 266 & USA & Caucasian & $10 \%$ INC & $H \& E$ & NA & $\begin{array}{l}\text { ypT0is } \\
\text { ypN0 }\end{array}$ & pCR & multivariate & $\mathrm{PMCb}, \mathrm{H}$ & $\begin{array}{l}\text { Neoadjuvant } \\
\text { setting }\end{array}$ \\
\hline $\begin{array}{l}\text { Kotoula } \\
\text { et al. }\end{array}$ & 2016 & retrospective & 333 & Greece & Caucasian & $>35 \%$ & $H \& E$ & NA & - & DFS & univariate & $\mathrm{A} / \mathrm{T}, \mathrm{H}$ & $\begin{array}{l}\text { Adjuvant } \\
\text { setting }\end{array}$ \\
\hline Liu et al. & 2015 & retrospective & 116 & China & Asian & $\geq 30 \%$ & $\mathrm{H} \& \mathrm{E}$ & $\begin{array}{l}33 \\
\text { months }\end{array}$ & $\begin{array}{l}\text { ypT0is } \\
\text { ypN0 }\end{array}$ & $\begin{array}{l}\text { pCR, } \\
\text { EFS }\end{array}$ & multivariate & $\mathrm{T} / \mathrm{Cb}, \mathrm{H}$ & $\begin{array}{l}\text { Neoadjuvant } \\
\text { setting }\end{array}$ \\
\hline $\begin{array}{l}\text { Perez et } \\
\text { al. }\end{array}$ & 2016 & prospective-retrospective & 456 & America & Caucasian & $10 \%$ INC & $H \& E$ & 4.4 years & - & RFS & multivariate & $\mathrm{DC}, \mathrm{P}, \mathrm{H}$ & $\begin{array}{l}\text { Adjuvant } \\
\text { setting }\end{array}$ \\
\hline Lee et al. & 2015 & retrospective & 447 & Korea & Asian & $10 \%$ INC & $H \& E$ & $\begin{array}{l}49 \\
\text { months }\end{array}$ & - & DFS & multivariate & $\begin{array}{l}\mathrm{AC}, \mathrm{P} / \mathrm{D}, \\
\mathrm{H}\end{array}$ & $\begin{array}{l}\text { Adjuvant } \\
\text { setting }\end{array}$ \\
\hline $\begin{array}{l}\text { Salgado } \\
\text { et al. }\end{array}$ & 2015 & retrospective & 250 & Australia & Caucasian & $\begin{array}{l}>5 \%, \\
1 \% \text { INC }\end{array}$ & $H \& E$ & 3.77 years & $\begin{array}{l}\text { ypT0is } \\
\text { ypN0 }\end{array}$ & $\begin{array}{l}\text { pCR } \\
\text { EFS }\end{array}$ & multivariate & $\mathrm{P}, \mathrm{H}, \mathrm{L}$ & $\begin{array}{l}\text { Neoadjuvant } \\
\text { setting }\end{array}$ \\
\hline Luen et al. & 2017 & retrospective & 678 & multicenter & Mix & $10 \%$ INC & $H \& E$ & $\begin{array}{l}50 \\
\text { months }\end{array}$ & - & PFS & multivariate & $\begin{array}{l}\mathrm{H}, \\
\mathrm{D}, \mathrm{Pe} / \mathrm{Pl}\end{array}$ & $\begin{array}{l}\text { Adjuvant } \\
\text { setting }\end{array}$ \\
\hline $\begin{array}{l}\text { Heppner } \\
\text { et al. }\end{array}$ & 2016 & retrospective & 340 & multicenter & Mix & $10 \%$ INC & $\mathrm{H} \& \mathrm{E}$ & NA & $\begin{array}{l}\text { ypT0is } \\
\text { ypN0 }\end{array}$ & pCR & multivariate & $\begin{array}{l}\mathrm{EC}, \\
\mathrm{D} / \mathrm{Cb}, \mathrm{H}\end{array}$ & $\begin{array}{l}\text { Neoadjuvant } \\
\text { setting }\end{array}$ \\
\hline Liu et al. & 2016 & retrospective & 101 & China & Asian & NA & $\mathrm{IHC}$ & NA & $\begin{array}{l}\text { ypT0is } \\
\text { ypN0 }\end{array}$ & $\mathrm{pCR}$ & multivariate & $\mathrm{TC}, \mathrm{H}$ & $\begin{array}{l}\text { Neoadjuvant } \\
\text { setting }\end{array}$ \\
\hline Dieci et al. & 2016 & prospective & 32 & USA & Caucasian & $1 \%$ INC & $H \& E$ & NA & $\begin{array}{l}\text { ypT0is } \\
\text { ypN0 }\end{array}$ & $\mathrm{pCR}$ & NA & $\mathrm{P}, \mathrm{FEC}, \mathrm{H}$ & $\begin{array}{l}\text { Neoadjuvant } \\
\text { setting }\end{array}$ \\
\hline
\end{tabular}

Abbreviations: H\&E, Hematoxylin-eosin staining; $10 \%$ INC, $10 \%$ increment; LPBC, lymphocyte-predominant breast cancer: $\geqq 60 \%$; pCR, pathological complete response; DFS, disease-free survival; DDFS, distant disease-free survival; EFS, event-free survival; RFS, recurrence-free survival; PFS, progression-free survival; OS, overall survival; NA, not available; AC, anthracycline-cyclophosphamide; P, paclitaxel; DC, doxorubicin-cyclophosphamide; D, docetaxel; FEC, fluorouracil/epirubicin/cyclophosphamide; $\mathrm{T}$, taxanes; $\mathrm{Cb}$, carboplatin; Pe, pertuzumab; Pl, placebo; L, lapatinib; PMCb, paclitaxel/doxorubicin/capecitabine; TC, taxanes-cyclophosphamide; $\mathrm{H}$, trastuzumab. 


\section{Effects of TILs on survival outcome}

As mentioned above, EFS was considered as the final uniform prognostic outcome. Seven studies assessed the prognostic value of trastuzumab-based treatment in HER2-positive BC patients upon different levels of TILs. Due to the heterogeneity among studies was not significant $\left(p>0.05, I^{2}=\right.$ $34.0 \%)$, the fixed-effects model was performed. The pooled results showed that high level of TILs was associated with improved EFS $(\mathrm{HR}=0.97 ; 95 \% \mathrm{CI}=$ 0.96-0.99, $P_{\mathrm{H}}=0.168$; Figure 2A), indicating that elevated TILs had prognostic value on the trastuzumab-based treatment outcomes in HER2-positive BC patients. As shown in Table 2, the stratified analysis revealed that high level of TILs was associated with better outcome in the subgroup of studies designed as retrospective $(\mathrm{HR}=0.97 ; 95 \% \mathrm{CI}=$ 0.96-0.99, $\left.P_{\mathrm{H}}=0.098\right)$, Caucasian population $(\mathrm{HR}=$ $\left.0.97 ; 95 \% \mathrm{CI}=0.95-0.99, P_{\mathrm{H}}=0.258\right), 10 \%$ INC cut-off value $\left(\mathrm{HR}=0.98 ; 95 \% \mathrm{CI}=0.96-0.99, P_{\mathrm{H}}=0.503\right)$. Additionally, five studies were pooled for analysis of the effects of TILs on survival outcome by multivariate analysis and the summarized results indicated that the level of TILs was an independent prognostic marker for better outcome $(\mathrm{HR}=0.97 ; 95 \%$
$\left.\mathrm{CI}=0.96-0.99, \quad P_{\mathrm{H}}=0.213\right)$ in studies enrolled HER2-positive BC patients receiving trastuzumab-based treatment.

\section{Effects of TILs on pCR}

Six studies were pooled for analysis of the effects of TILs on $\mathrm{pCR}$ and the main results were listed in Table 2 and Figure 2B. Due to the heterogeneity among studies was significant $\left(p<0.05, I^{2}=87.9 \%\right)$, the random-effects model was performed. The results indicated that elevated TILs predicted a higher $\mathrm{pCR}$ rate for the HER2-positive $\mathrm{BC}$ patients with trastuzumab-based treatment $(\mathrm{OR}=1.32 ; 95 \% \mathrm{CI}=$ 1.10-1.60, $\left.P_{\mathrm{H}}<0.001\right)$. Subgroup analysis showed that high level of TILs was associated with improved pCR rate in the subgroup of the retrospective study (OR = $\left.2.71 ; 95 \% \mathrm{CI}=1.12-6.53, P_{\mathrm{H}}<0.001\right)$, Asian population $\left(\mathrm{OR}=4.98 ; 95 \% \mathrm{CI}=2.59-9.59, P_{\mathrm{H}}=0.883\right), 10 \% \mathrm{INC}$ cut-off value $\left(\mathrm{OR}=1.18 ; 95 \% \mathrm{CI}=1.10-1.28, P_{\mathrm{H}}=\right.$ $0.086)$ and other cut-off value subgroup $(\mathrm{OR}=2.67$; $\left.95 \% \mathrm{CI}=1.04-6.83, P_{\mathrm{H}}<0.001\right)$. However, no significant association between elevated TILs and higher $\mathrm{pCR}$ rate was observed in the subgroup of prospective study $\left(\mathrm{OR}=1.14 ; 95 \% \mathrm{CI}=0.92-1.41, P_{\mathrm{H}}<\right.$ $0.001)$ and Caucasian population $(\mathrm{OR}=1.23 ; 95 \% \mathrm{CI}=$ $\left.0.97-1.56, P_{\mathrm{H}}<0.001\right)$.

Table 2. The main results of pooled studies

\begin{tabular}{|c|c|c|c|c|c|c|c|c|}
\hline \multirow[t]{2}{*}{ Outcome } & \multirow[t]{2}{*}{ Variables } & \multirow[t]{2}{*}{ Number of studies } & \multirow[t]{2}{*}{ Number of patients } & \multicolumn{3}{|c|}{$P$-value } & \multicolumn{2}{|c|}{ Regression model } \\
\hline & & & & $\mathrm{P}_{\mathrm{H}}$ & $\mathrm{P}_{\mathrm{Z}}$ & $P_{E}$ & Random & Fixed \\
\hline \multirow[t]{14}{*}{ EFS } & ALL & 7 & 2489 & 0.168 & 0.000 & 0.043 & $0.97(0.95,0.99)$ & $0.97(0.96,0.99)^{*}$ \\
\hline & Study design & & & & & & & \\
\hline & Retrospective & 5 & 1824 & 0.098 & 0.000 & - & $0.97(0.96,1.00)$ & $0.97(0.96,0.99) *$ \\
\hline & Prospective-retrospective & 2 & 665 & 0.269 & 0.805 & - & $0.97(0.83,1.14)$ & $0.99(0.87,1.11)$ \\
\hline & Ethnicity & & & & & & & \\
\hline & Caucasian & 3 & 1039 & 0.258 & 0.004 & - & $0.98(0.91,1.05)$ & $0.97(0.95,0.99) *$ \\
\hline & Asian & 2 & 563 & 0.039 & 0.415 & - & $0.54(0.13,2.35)$ & $0.98(0.96,1.00)$ \\
\hline & Mix & 2 & 887 & 0.411 & 0.040 & - & $0.95(0.90,1.00)$ & $0.95(0.90,1.00)$ \\
\hline & Cut-off value & & & & & & & \\
\hline & $10 \% \mathrm{INC}^{+}$ & 4 & 1790 & 0.503 & 0.007 & - & $0.98(0.96,0.99)$ & $0.98(0.96,0.99) *$ \\
\hline & Other $^{+}$ & 3 & 699 & 0.038 & 0.199 & - & $0.44(0.13,1.53)$ & $0.97(0.95,0.99)$ \\
\hline & Analysis method & & & & & & & \\
\hline & Univariate & 2 & 542 & 0.180 & 0.169 & - & $0.59(0.19,1.85)$ & $0.79(0.56,1.11)$ \\
\hline & Multivariate & 5 & 1947 & 0.213 & 0.000 & - & $0.97(0.95,0.99)$ & $0.97(0.96,0.99)^{*}$ \\
\hline \multirow[t]{11}{*}{ pCR } & ALL & 6 & 1105 & 0.000 & 0.004 & 0.001 & $1.32(1.10,1.60)^{*}$ & $1.06(1.03,1.09)$ \\
\hline & Study design & & & & & & & \\
\hline & Prospective & 2 & 298 & 0.000 & 0.228 & - & $1.14(0.92,1.41)$ & $1.05(1.01,1.08)$ \\
\hline & Retrospective & 4 & 807 & 0.000 & 0.027 & - & $2.71(1.12,6.53)^{*}$ & $1.17(1.07,1.29)$ \\
\hline & Ethnicity & & & & & & & \\
\hline & Caucasian & 3 & 548 & 0.000 & 0.091 & - & $1.23(0.97,1.56)$ & $1.05(1.02,1.09)$ \\
\hline & Asian & 2 & 217 & 0.883 & 0.000 & - & $4.98(2.59,9.59)$ & $4.98(2.59,9.59)^{*}$ \\
\hline & Mix & 1 & 340 & - & 0.023 & - & $1.12(1.02,1.24)$ & $1.12(1.02,1.24)^{*}$ \\
\hline & Cut-off value & & & & & & & \\
\hline & $10 \% \mathrm{INC}^{+}$ & 2 & 606 & 0.086 & 0.000 & - & $1.19(1.05,1.36)$ & $1.18(1.10,1.28)^{*}$ \\
\hline & Other $^{+}$ & 4 & 499 & 0.000 & 0.041 & - & $2.67(1.04,6.83)^{*}$ & $1.04(1.00,1.07)$ \\
\hline
\end{tabular}

The bold and "*" represent that OR or HR with corresponding 95\% CI was used to analyze and was statistically significant results, respectively.

"10\% INC" group means the cut-off value of TILs is per $10 \%$ increment, and "Other" ${ }^{\prime \prime}$ group means a set of other different cut-off values of TILs. $P_{\mathrm{H}}, P$-value of heterogeneity test; $P_{\mathrm{z}}, P$-value of $t$-test; $P_{\mathrm{E}}, P$-value of Egger's test. 
A

Study

ID

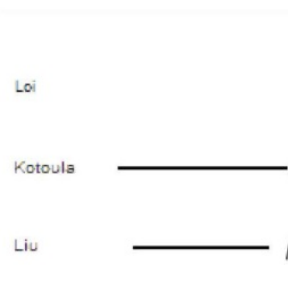

Perez

Lee

Saigado

Luen

Overall (1-squared $=34.0 \%, p=0.188$ )

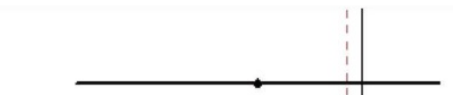

II

$\|$

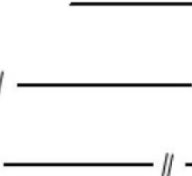

II

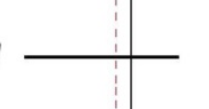

$\|$

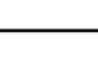

HR $(95 \% \mathrm{Cl})$

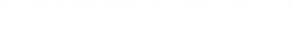

B

Study

ID

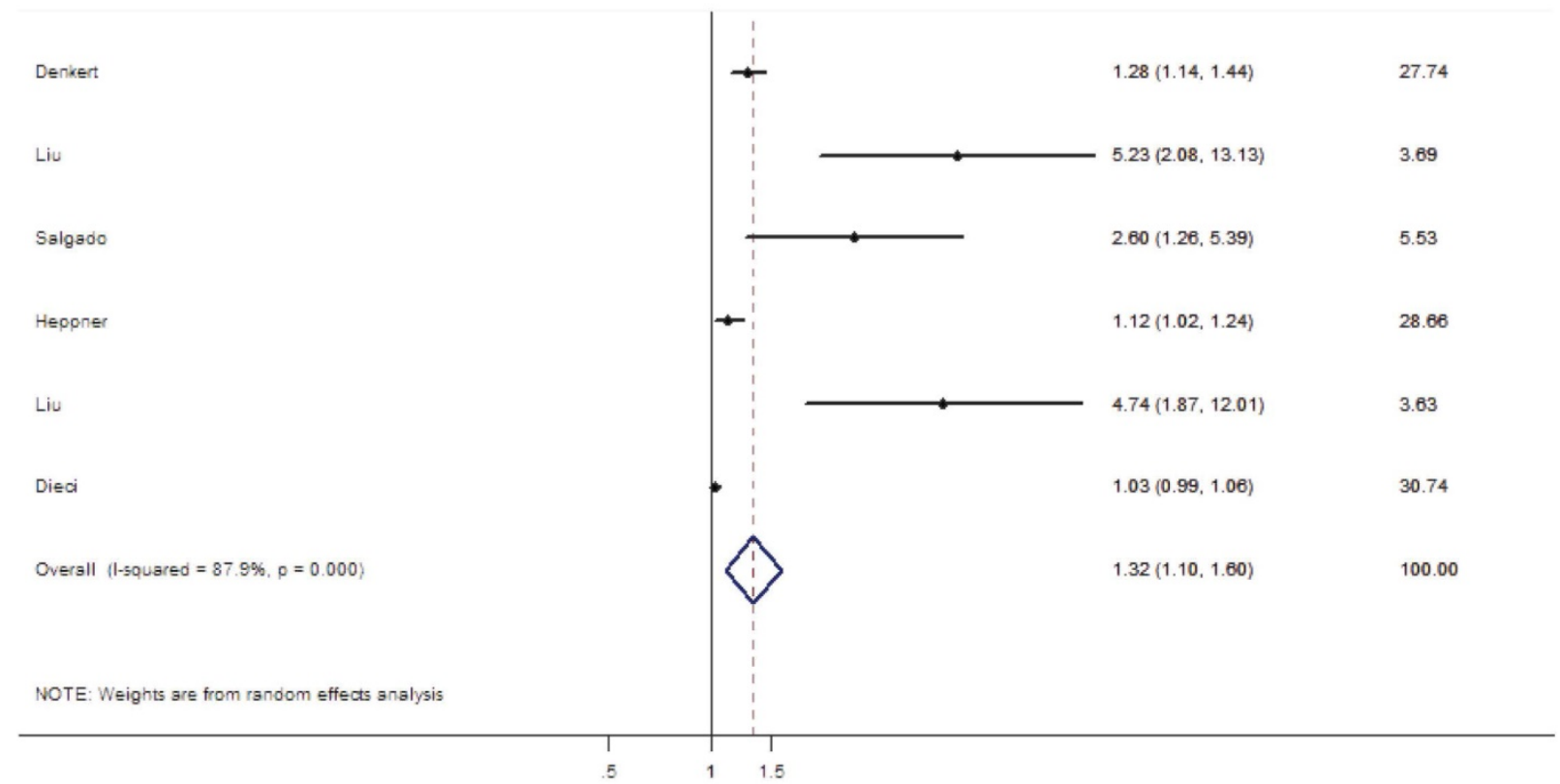

Figure 2. Forest plots indicating the predictive and prognostic role of TILs in HER2-positive BC patients with trastuzumab-based treatment. (A) EFS (fixed-effects model); (B) PCR (random-effects model). 


\section{Sensitivity analysis}

Sensitivity analysis was carried out to assess the influence of each individual study and sources of heterogeneity on the summary effect for pCR and EFS through repeating the meta-analysis after sequentially omitting each study. As shown in the Figure 3A, the pooled result for EFS did not change when deleting one study, indicating our result was stable and robust.
However, for $\mathrm{pCR}$, the sensitivity analysis suggested that studies by Heppner et al. [23] and Dieci et al. [37] were the main origin heterogeneity (Figure 3B). Even so, after omitting these two studies respectively, the results still supported our previous conclusion (OR $=$ $1.61,95 \% \mathrm{CI}=1.19-2.19$; $\mathrm{OR}=1.65,95 \% \mathrm{CI}=1.23-2.22$, respectively).

A

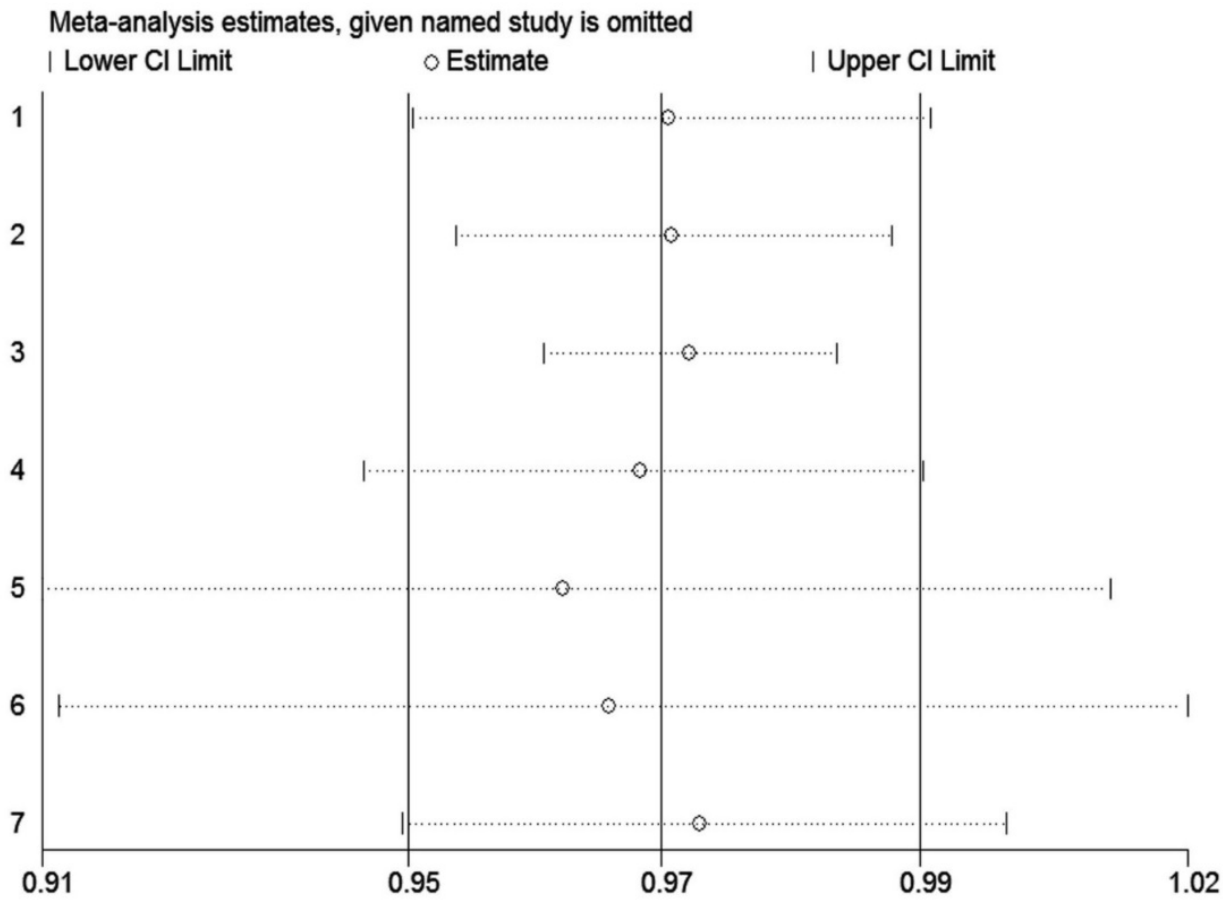

B

Meta-analysis estimates, given named study is omitted | Lower Cl Limit

o Estimate

| Upper Cl Limit

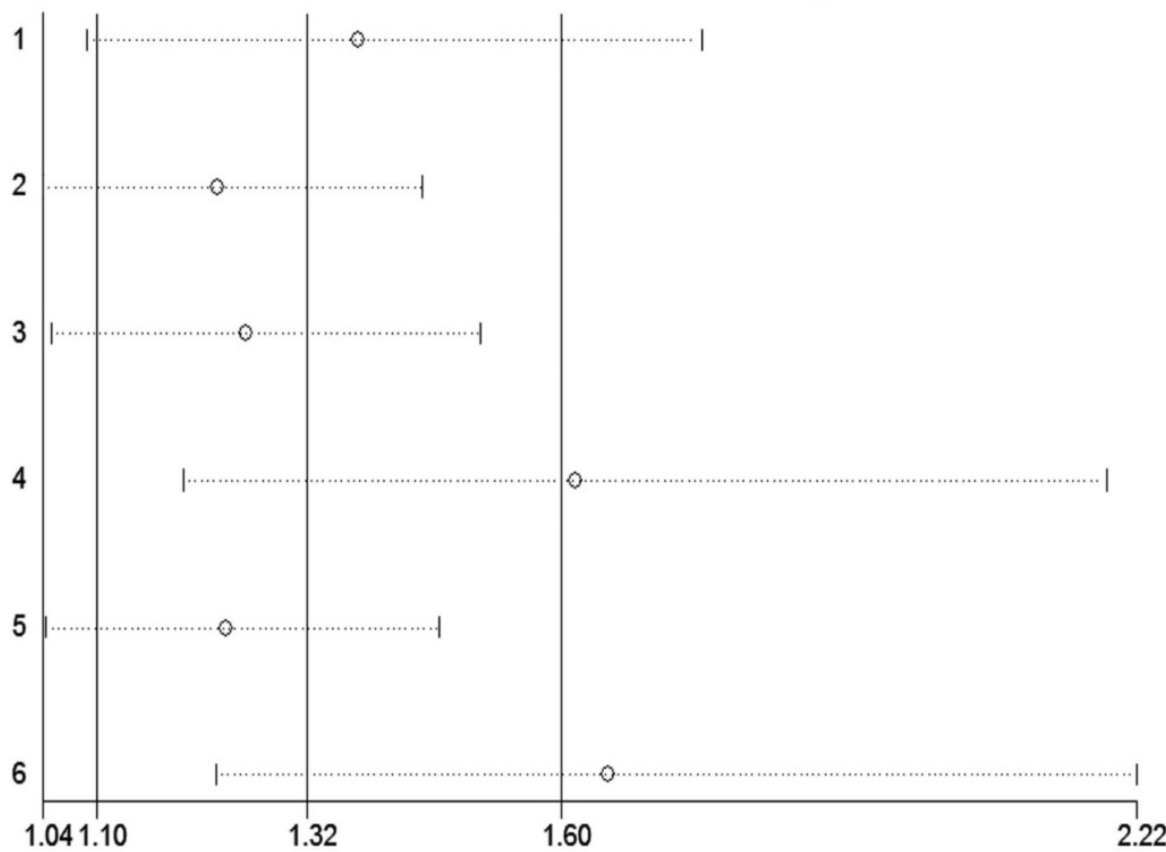

Figure 3. Sensitivity analysis of studies included in this meta-analysis. (A) EFS; (B) PCR 

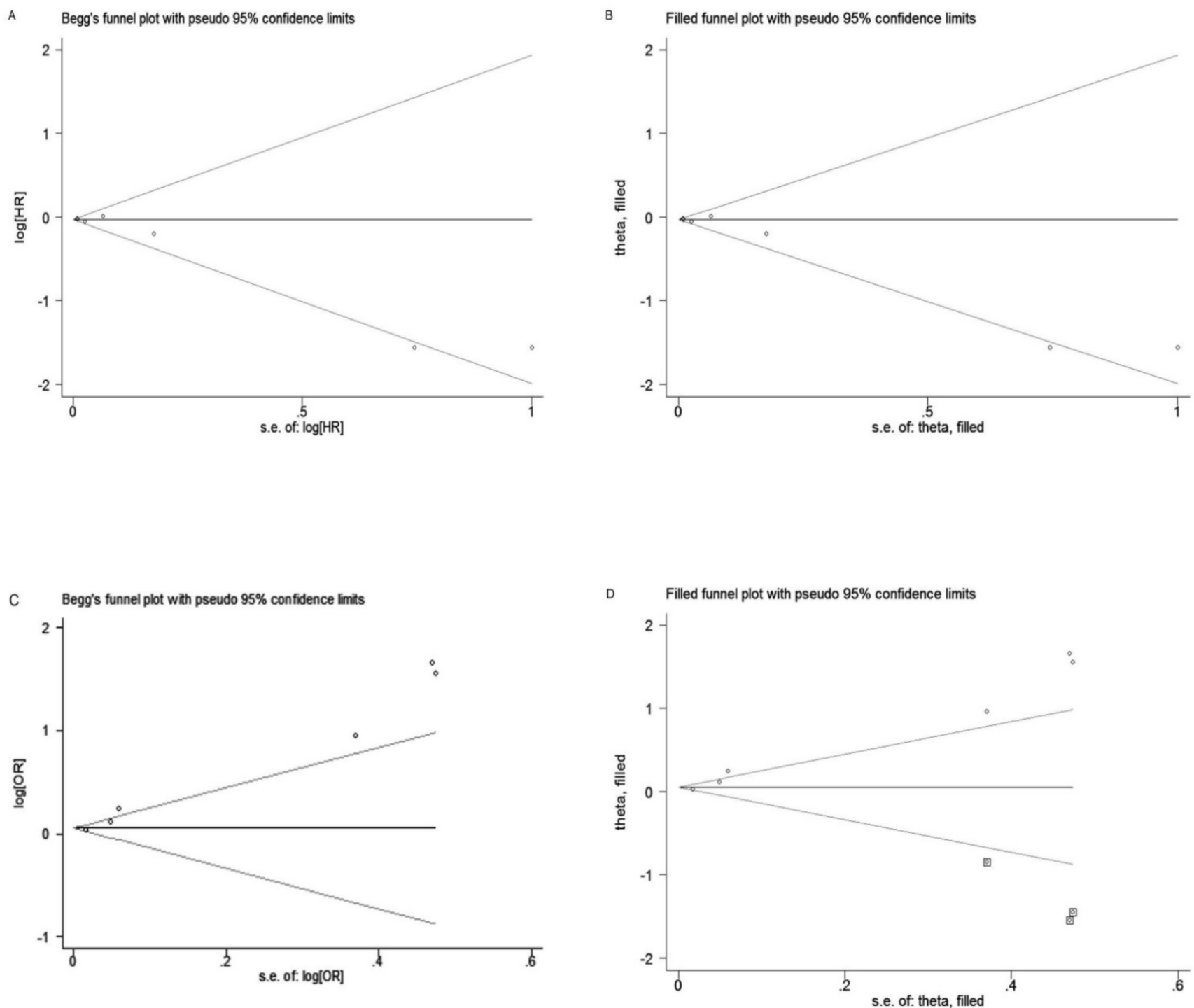

Figure 4. Funnel plot and trim-and-fill analysis of studies included in this meta-analysis. (A) EFS; (B) EFS after trim-and-fill analysis; (C) $p C R$; (D) $p C R$ after trim-and-fill analysis.

\section{Publication bias}

Begg's test and Egger's test were performed to assess the publication bias. The shape of funnel plot for EFS was slight asymmetry and Egger's test showed negligible publication bias $\left(P_{\mathrm{B}}=0.035, P_{\mathrm{E}}=\right.$ 0.043; Figure $4 \mathrm{~A})$. However, for $\mathrm{pCR}$, the shape of funnel plot was obviously asymmetry and Egger's test also provided statistical evidence of funnel plot asymmetry $\left(P_{\mathrm{B}}=0.133, P_{\mathrm{E}}=0.001\right.$; Figure $\left.4 \mathrm{C}\right)$. To adjust the bias, a trim-and-fill analysis method reported by Duval and Tweedie [46] was performed, which indicating that for EFS the conclusion before and after was not changed, suggesting the results was stable (Figure 4B). However, for pCR, three studies need to be filled (Figure 4D).

\section{Discussion}

The current meta-analysis incorporating 11 eligible relevant studies containing 3228 BC patients has revealed that high level of TILs was significantly associated with increased pCR rates and improved outcomes of patients, indicating a predictive and prognostic role of TILs in HER2-positive BC patients with trastuzumab-based treatment.

In this paper, we observed that elevated TILs were associated with higher $\mathrm{pCR}$ rates and improved outcomes in all the included population and in the subgroup of retrospective study design and $10 \%$ INC cut-off value but not in the subgroup of prospective or prospective-retrospective study design and other cut-off value. For the study design subgroup, the majority of study design of the included studies was retrospective studies and due to the accuracy of collected data, retrospective studies often have substantial advantages to biomarker evaluation. For the cut-off value subgroup, $10 \%$ INC was frequently used in most articles and considered as an effective 
cut-off value by many investigators. The result in the $10 \%$ INC subgroup was consistent with the overall result, while the result in the other cut-off value subgroup was not, suggesting that $10 \%$ INC cut-off value was appropriate to evaluate the role of TILs, which was also consistent with the conclusion from the meta-analysis by Mao et al. [24]. For the ethnicity subgroup, the results indicated that elevated TILs predicted higher $\mathrm{pCR}$ rates in the Asian population and better survival in the Caucasian population, respectively. We observed that the heterogeneity was significant in the Caucasian population for $\mathrm{pCR}$ and in the Asian population for survival outcome, so the random-effects model was performed. And due to the fewer studies in these subgroups, it may not provide statistical power to show significant result. However, the results in the fix-effects model were consistent with the overall result, as shown in Table 2. With the number of enrolled studies increasing, the heterogeneity could decrease and significant results could be obtained from the fix-effects model.

As far as we know, HER-2 expression in the BC tissue has been considered as a molecular marker for the treatment. Trastuzumab as targeted drug that blocks HER2 in HER2-positive BC by binding to the extracellular domain has been applied and proved with remarkable clinical efficacy for the BC patients with HER2-positive expression [47]. However, a challenge that low response rate and development of drugs resistance was emerged along with the continued trastuzumab-based treatment. The potential mechanism was partially the loss of expression of HER2 extracellular domain on tumor cells leading to the ineffectiveness of trastuzumab. Previous studies have concluded that HER2 overexpression could promote epithelial to mesenchymal transition (EMT), and increased expression and activation of metalloproteinases during EMT could lead to proteolytic cleavage and shedding of HER2 receptor, which downregulates HER2 extracellular domain and eventually increases trastuzumab resistance [48]. Additionally, studies have also revealed that trastuzumab resistance could be triggered by the excessive activation of oncogenic signaling pathways including PI3K/AKT and MARK pathway $[49,50]$. Importantly, the immune system plays an essential role for the trastuzumab effect. It is well known that immunosuppression could contribute to the tumor progression and treatment resistance [51]. It has also been reported that the antitumor effects of cytotoxic regimens and antibody-based therapy could be enhanced through maintaining the stability of the immune systems, which was observed in the animal model that the effect of trastuzumab was significantly decreased when treating immune-deficient mice [19] and stimulation of immune system could enhance the therapeutic effect [20]. TILs as an important component of immune system could release immunosuppression [7] and was regarded as an effective biomarker of host anti-tumor immune response in the tumor microenvironment, which have great contribution to the efficacy of trastuzumab therapy. Therefore, TILs in this study has been discussed as a prognostic marker for the BC patients receiving trastuzumab-based treatment. As mentioned above, the patients enrolled in our study were all received trastuzumab treatment targeted the HER-2 molecule, although the participants in the studies by Salgado et al. [32] and Luen et al. [36] received lapatinib and pertuzumab respectively in addition to trastuzumab, there was no significant interaction between TILs and the combination group of trastuzumab and lapatinib versus (vs.) trastuzumab alone in predicting pCR and EFS [32]. Similarly, the effect of the addition of pertuzumab and association between TILs and clinical outcome was not observed [36]. Additionally, there is little research on the association between TILs and lapatinib and pertuzumab. Therefore, we have reasons to believe that the above two anti-HER2 agents do not affect the relationship between TILs and trastuzumab. In these clinical researches, another important factor influencing the results was considerable heterogeneity in the chemotherapy treatment regimens. The combined use of chemotherapy may mask the true impact of TILs on response to trastuzumab. All studies in the analysis were combined chemotherapy and trastuzumab. However, almost all results were obtained after adjusting for age, ethnicity, chemotherapy regimen and other factors, indicating that the level of TILs was an independent predictive and prognostic marker for pCR and survival in HER2-positive BC patients with trastuzumab-based treatment. The above evidence supported our result that TILs were emerging as a robust predictive biomarker of trastuzumab benefit in HER2-positive BC. In order to exactly investigate the relationship between TILs and trastuzumab benefit, further studies with large patient samples need to be conducted.

Although the current meta-analysis has provided sufficient useful information concerning the predictive and prognostic role of stromal TILs in HER2-positive BC with trastuzumab-based treatment, there still remains some limitations. First, although most of the included studies have adopted H\&E staining methods to determine the quantities of TILs, there might be a difference when assessing the presence of TILs in a surgical specimen because it is a 
semiquantitative maker. Second, the cut-off values of TILs varied in our analysis, which may partly influence the pooled results of the pCR and survival analysis while we have conducted a subgroup analysis based on different cut-off values. Therefore, accurate detection method and unified quantification criteria of TILs value should be performed in further studies. Third, the outcome events in the included studies were different and we determined EFS as a uniform standard for prognostic outcome because EFS contained every other study endpoint, which might have impacted the validity of survival analysis. Therefore, the further studies focused on uniform outcome event with large sample size should be conducted in order to evaluate the prognostic role of TILs more precisely. In addition, another potential limitation was obvious publication bias in $\mathrm{pCR}$ analysis. Due to the fact that studies obtaining positive results could be more easily published than studies with negative results and studies included in our analysis was fewer, it may lead to some data missing and publication bias.

In conclusion, the present meta-analysis proved a significant association between TILs and trastuzumab benefit, indicating the predictive and prognostic role of stromal TILs in HER2-positive BC receiving trastuzumab-based treatment. However, with limitations of the study, multi-center and well-designed prospective studies with large sample size are needed to further validate our findings.

\section{Abbreviations}

TILs, tumor-infiltrating lymphocytes; BC, breast cancer; pCR, pathologic complete response; DFS, disease-free survival; DDFS, distant disease-free survival; EFS, event-free survival; RFS, recurrence-free survival; PFS, progression-free survival; OR, odds ratio; $\mathrm{HR}$, hazard ratio; $95 \% \mathrm{CI}$, $95 \%$ confidence interval; $P_{\mathrm{H}}, P$-value of heterogeneity.

\section{Acknowledgments}

This project was supported by grants from the National Nature Science Foundation of China (No.81472027 to S.W and No.81501820 to Y.P); Jiangsu Provincial Medical Youth Talent to B.H (QNRC2016066) and Y.P (QNRC2016074); Nanjing Medical Science and Technique Development Foundation to B.H (JQX13003) and Nanjing Medical University Science and Technique Development Foundation Project to HL. S (No.2015NJMUZD049).

\section{Authors' contributions}

$\mathrm{BSH}$ and YQP participated in the design of this study, TX and XXL acquired the data and performed the analysis, XXH and XXC contributed to the data analysis, KL and HXP checked the results, BSH and HLS revised the draft, TX and SKW drafted the paper.

\section{Competing Interests}

The authors have declared that no competing interest exists.

\section{References}

1. Siegel RL, Miller KD, Jemal A. Cancer Statistics, 2017. CA: a cancer journal for clinicians. 2017; 67: 7-30.

2. Slamon DJ, Clark GM, Wong SG, et al. Human breast cancer: correlation of relapse and survival with amplification of the HER-2/neu oncogene. Science. 1987; 235: 177-82.

3. Slamon DI, Godolphin W, Jones LA, et al Studies of the HER-2/neu proto-oncogene in human breast and ovarian cancer. Science. 1989; 244 : 707-12.

4. Kiessling R, Wei WZ, Herrmann F, et al. Cellular immunity to the Her-2/neu protooncogene. Advances in cancer research. 2002; 85: 101-44.

5. Kast K, Schoffer O, Link T, et al. Trastuzumab and survival of patients with metastatic breast cancer. Archives of gynecology and obstetrics. 2017; 296: 303-12.

6. Pruneri G, Vingiani A, Denkert C. Tumor infiltrating lymphocytes in early breast cancer. Breast. 2017.

7. Dushyanthen S, Beavis PA, Savas P, et al. Relevance of tumor-infiltrating lymphocytes in breast cancer. BMC medicine. 2015; 13: 202.

8. Pardoll DM. The blockade of immune checkpoints in cancer immunotherapy. Nature reviews Cancer. 2012; 12: 252-64

9. Denkert C, Loibl S, Noske A, et al. Tumor-associated lymphocytes as an independent predictor of response to neoadjuvant chemotherapy in breast cancer. Journal of clinical oncology : official journal of the American Society of Clinical Oncology. 2010; 28: 105-13.

10. Kashiwagi S, Asano Y, Goto W, et al. Use of Tumor-infiltrating lymphocytes (TILs) to predict the treatment response to eribulin chemotherapy in breast cancer. PloS one. 2017; 12: e0170634.

11. Loi S, Sirtaine N, Piette F, et al. Prognostic and predictive value of tumor-infiltrating lymphocytes in a phase III randomized adjuvant breast cancer trial in node-positive breast cancer comparing the addition of docetaxel to doxorubicin with doxorubicin-based chemotherapy: BIG 02-98. Journal of clinical oncology : official journal of the American Society of Clinical Oncology. 2013; 31: 860-7.

12. Aaltomaa S, Lipponen $\mathrm{P}$, Eskelinen $\mathrm{M}$, et al. Lymphocyte infiltrates as a prognostic variable in female breast cancer. European journal of cancer. 1992; 28A: 859-64.

13. Adams S, Gray RJ, Demaria S, et al. Prognostic value of tumor-infiltrating lymphocytes in triple-negative breast cancers from two phase III randomized adjuvant breast cancer trials: ECOG 2197 and ECOG 1199. Journal of clinical oncology : official journal of the American Society of Clinical Oncology. 2014; 32: 2959-66.

14. Rathore AS, Kumar S, Konwar R, et al. CD3+, CD4+ \& CD8+ tumour infiltrating lymphocytes (TILs) are predictors of favourable survival outcome in infiltrating ductal carcinoma of breast. The Indian journal of medical research. 2014; 140:361-9.

15. Okabe M, Toh U, Iwakuma N, et al. Predictive factors of the tumor immunological microenvironment for long-term follow-up in early stage breast cancer. Cancer science. 2017; 108: 81-90.

16. Apetoh L, Ghiringhelli F, Tesniere A, et al. Toll-like receptor 4-dependent contribution of the immune system to anticancer chemotherapy and radiotherapy. Nature medicine. 2007; 13: 1050-9.

17. Mattarollo SR, Loi S, Duret $\mathrm{H}$, et al. Pivotal role of innate and adaptive immunity in anthracycline chemotherapy of established tumors. Cancer research. 2011; 71: 4809-20.

18. Varadan V, Gilmore H, Miskimen KL, et al. Immune Signatures Following Single Dose Trastuzumab Predict Pathologic Response to PreoperativeTrastuzumab and Chemotherapy in HER2-Positive Early Breast Cancer. Clinical cancer research : an official journal of the American Association for Cancer Research. 2016; 22: 3249-59.

19. Park S, Jiang Z, Mortenson ED, et al. The therapeutic effect of anti-HER2/neu antibody depends on both innate and adaptive immunity. Cancer cell. 2010; 18: $160-70$

20. Stagg J, Loi S, Divisekera U, et al. Anti-ErbB-2 mAb therapy requires type I and II interferons and synergizes with anti-PD-1 or anti-CD137 mAb therapy. Proceedings of the National Academy of Sciences of the United States of America. 2011: 108: 7142-7.

21. Savas P, Loi S. Investigating the positive relationship between tumor-infiltrating lymphocytes and trastuzumab therapy. Immunotherapy. 2014; 6: 803-5

22. Loi S, Michiels S, Salgado R, et al. Tumor infiltrating lymphocytes are prognostic in triple negative breast cancer and predictive for trastuzumab benefit in early breast cancer: results from the FinHER trial. Annals of 
oncology : official journal of the European Society for Medical Oncology / ESMO. 2014; 25: 1544-50

23. Ingold Heppner B, Untch $\mathrm{M}$, Denkert $\mathrm{C}$, et al. Tumor-Infiltrating Lymphocytes: A Predictive and Prognostic Biomarker in Neoadjuvant-Treated HER2-Positive Breast Cancer. Clinical cancer research : an official journal of the American Association for Cancer Research. 2016; 22: 5747-54.

24. Mao Y, Qu Q, Zhang Y, et al. The value of tumor infiltrating lymphocytes (TILs) for predicting response to neoadjuvant chemotherapy in breast cancer: a systematic review and meta-analysis. PloS one. 2014; 9: e115103.

25. Yu X, Zhang Z, Wang $Z$, et al. Prognostic and predictive value of tumor-infiltrating lymphocytes in breast cancer: a systematic review and meta-analysis. Clinical \& translational oncology : official publication of the Federation of Spanish Oncology Societies and of the National Cancer Institute of Mexico. 2016; 18: 497-506.

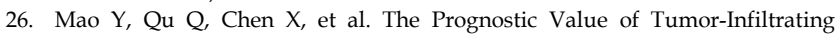
Lymphocytes in Breast Cancer: A Systematic Review and Meta-Analysis. PloS one. 2016; 11: e0152500.

27. Ibrahim EM, Al-Foheidi ME, Al-Mansour MM, et al. The prognostic value of tumor-infiltrating lymphocytes in triple-negative breast cancer: a meta-analysis. Breast cancer research and treatment. 2014; 148: 467-76.

28. Wang $\mathrm{K}, \mathrm{Xu}$ J, Zhang $\mathrm{T}$, et al. Tumor-infiltrating lymphocytes in breast cancer predict the response to chemotherapy and survival outcome: A meta-analysis. Oncotarget. 2016; 7: 44288-98

29. Denkert C, von Minckwitz G, Brase JC, et al. Tumor-infiltrating lymphocytes and response to neoadjuvant chemotherapy with or without carboplatin in human epidermal growth factor receptor 2-positive and triple-negative primary breast cancers. Journal of clinical oncology : official journal of the American Society of Clinical Oncology. 2015; 33: 983-91.

30. Liu S, Duan $X, X u$ L, et al. Optimal threshold for stromal tumor-infiltrating lymphocytes: its predictive and prognostic value in HER2-positive breast cancer treated with trastuzumab-based neoadjuvant chemotherapy. Breast cancer research and treatment. 2015; 154: 239-49.

31. Lee HJ, Kim JY, Park IA, et al. Prognostic Significance of Tumor-Infiltrating Lymphocytes and the Tertiary Lymphoid Structures in HER2-Positive Breast Cancer Treated With Adjuvant Trastuzumab. American journal of clinical pathology. 2015; 144: 278-88.

32. Salgado R, Denkert C, Campbell C, et al. Tumor-Infiltrating Lymphocytes and Associations With Pathological Complete Response and Event-Free Survival in HER2-Positive Early-Stage Breast Cancer Treated With Lapatinib and Trastuzumab: A Secondary Analysis of the NeoALTTO Trial. JAMA oncology. 2015; 1: 448-54.

33. Liu $\mathrm{S}, \mathrm{Xu} \mathrm{L}$, Zhang $\mathrm{H}$, et al. [Predictive factors of pathological complete response in primary human epidermal growth factor receptor 2-positive breast cancer treated with trastuzumab-based neoadjuvant chemotherapy]. Zhonghua wai ke za zhi [Chinese journal of surgery]. 2016; 54: 94-8.

34. Kotoula V, Chatzopoulos K, Lakis S, et al. Tumors with high-density tumor infiltrating lymphocytes constitute a favorable entity in breast cancer: a pooled analysis of four prospective adjuvant trials. Oncotarget. 2016; 7: 5074-87.

35. Perez EA, Ballman KV, Tenner KS, et al. Association of Stromal Tumor-Infiltrating Lymphocytes With Recurrence-Free Survival in the N9831 Adjuvant Trial in Patients With Early-Stage HER2-Positive Breast Cancer. JAMA oncology. 2016; 2: 56-64.

36. Luen SJ, Salgado R, Fox S, et al. Tumour-infiltrating lymphocytes in advanced HER2-positive breast cancer treated with pertuzumab or placebo in addition to trastuzumab and docetaxel: a retrospective analysis of the CLEOPATRA study. The Lancet Oncology. 2017; 18: 52-62.

37. Dieci MV, Prat A, Tagliafico E, et al. Integrated evaluation of PAM50 subtypes and immune modulation of pCR in HER2-positive breast cancer patients treated with chemotherapy and HER2-targeted agents in the CherLOB trial. Annals of oncology : official journal of the European Society for Medical Oncology / ESMO. 2016; 27: 1867-73.

38. Knobloch K, Yoon U, Vogt PM. Preferred reporting items for systematic reviews and meta-analyses (PRISMA) statement and publication bias. Journal of cranio-maxillo-facial surgery : official publication of the European Association for Cranio-Maxillo-Facial Surgery. 2011; 39: 91-2.

39. Moher D, Liberati A, Tetzlaff J, et al. Preferred reporting items for systematic reviews and meta-analyses: the PRISMA statement. Journal of clinical epidemiology. 2009; 62: 1006-12.

40. Handoll HH. Systematic reviews on rehabilitation interventions. Archives of physical medicine and rehabilitation. 2006; 87: 875.

41. Higgins JP, Thompson SG, Deeks JJ, et al. Measuring inconsistency in meta-analyses. Bmj. 2003; 327: 557-60.

42. DerSimonian R, Laird N. Meta-analysis in clinical trials. Controlled clinical trials. 1986; 7: 177-88.

43. Mantel N, Haenszel W. Statistical aspects of the analysis of data from retrospective studies of disease. Journal of the National Cancer Institute. 1959; 22: 719-48.

44. Egger M, Davey Smith G, Schneider M, et al. Bias in meta-analysis detected by a simple, graphical test. Bmj. 1997; 315: 629-34.

45. Begg CB, Mazumdar M. Operating characteristics of a rank correlation test for publication bias. Biometrics. 1994; 50: 1088-101

46. Duval S, Tweedie R. Trim and fill: A simple funnel-plot-based method of testing and adjusting for publication bias in meta-analysis. Biometrics. 2000; 56: 455-63.
47. von Minckwitz G, Procter M, de Azambuja E, et al. Adjuvant Pertuzumab and Trastuzumab in Early HER2-Positive Breast Cancer. The New England journal of medicine. 2017; 377: 122-31.

48. Nami B, Wang Z. HER2 in Breast Cancer Stemness: A Negative Feedback Loop towards Trastuzumab Resistance. Cancers. 2017; 9.

49. Nelson JM, Fry DW. Akt, MAPK (Erk1/2), and p38 act in concert to promote apoptosis in response to ErbB receptor family inhibition. The Journal of biological chemistry. 2001; 276: 14842-7.

50. Hernandez-Aya LF, Gonzalez-Angulo AM. Targeting the phosphatidylinositol 3-kinase signaling pathway in breast cancer. The oncologist. 2011; 16: 404-14.

51. Criscitiello C, Curigliano G. Immunotherapy of Breast Cancer. Progress in tumor research. 2015; 42: 30-43. 